\title{
A functional genetic toolbox for human tissue-derived organoids
}

\author{
Dawei Sun 1 , Lewis Evans², Francesca Perrone ${ }^{3}$, Vanesa Sokleva ${ }^{3}$, Kyungtae Lim 1 , \\ Saba Rezakhani ${ }^{4}$, Matthias Lutolf ${ }^{4}$, Matthias Zilbauer ${ }^{3}$, Emma L Rawlins ${ }^{3 *}$ \\ ${ }^{1}$ Wellcome Trust/CRUK Gurdon Institute, University of Cambridge, Cambridge, \\ United Kingdom; ${ }^{2}$ Developmental Biology and Cancer Development, University \\ College London, London, United Kingdom; ${ }^{3}$ University of Cambridge, Cambridge, \\ United Kingdom; ${ }^{4}$ École Polytechnique Fédérale de Lausanne (EPFL), Lausanne, \\ Switzerland
}

*For correspondence: elr21@cam.ac.uk

Competing interest: The authors declare that no competing interests exist.

Funding: See page 14

Preprinted: 05 May 2020 Received: 03 March 2021

Accepted: 05 October 2021

Published: 06 October 2021

Reviewing Editor: Marianne E Bronner, California Institute of Technology, United States

(c) Copyright Sun et al. This article is distributed under the terms of the Creative Commons Attribution License, which permits unrestricted use and redistribution provided that the original author and source are credited.

\begin{abstract}
Human organoid systems recapitulate key features of organs offering platforms for modelling developmental biology and disease. Tissue-derived organoids have been widely used to study the impact of extrinsic niche factors on stem cells. However, they are rarely used to study endogenous gene function due to the lack of efficient gene manipulation tools. Previously, we established a human foetal lung organoid system (Nikolić et al., 2017). Here, using this organoid system as an example, we have systematically developed and optimised a complete genetic toolbox for use in tissue-derived organoids. This includes 'Organoid Easytag', our efficient workflow for targeting all types of gene loci through CRISPR-mediated homologous recombination followed by flow cytometry for enriching correctly targeted cells. Our toolbox also incorporates conditional gene knockdown or overexpression using tightly inducible CRISPR interference and CRISPR activation which is the first efficient application of these techniques to tissue-derived organoids. These tools will facilitate gene perturbation studies in tissue-derived organoids facilitating human disease modelling and providing a functional counterpart to many ongoing descriptive studies, such as the Human Cell Atlas Project.
\end{abstract}

\section{Main}

CRISPR and its related techniques (CRISPR interference [CRISPRi] and CRISPR activation [CRISPRa]) have transformed the study of gene function in model systems. They have been rapidly adopted in cancer and pluripotent stem cell (PSC) lines (Bowden et al., 2020; Gilbert et al., 2013; Tian et al., 2019), but not in tissue-derived human organoids. We have optimised these genetic tools for use in organoids using a tissue-derived human foetal lung organoid system (Nikolić et al., 2017).

First, we aimed to establish a robust workflow for gene targeting in organoids to facilitate reporter and direct knockout (KO) generation. A recent application of non-homologous end joining (NHEJ) to improve gene targeting in organoids has been successful (Artegiani et al., 2020). However, we adopted a homology-directed repair (HDR) strategy as a complementary approach. We reasoned that the recombination-based method allows our strategy to deliver precise genetic manipulation with flexible targeting sites and minimal additional genetic changes. We chose fluorescence as a selection marker, allowing targeted cells to be easily isolated using flow cytometry and chimeric colonies to be identified and removed using a fluorescent microscope.

To achieve efficient gene targeting, we first sought to maximise (1) the efficiency of DNA delivery into organoid cells and (2) the efficiency of site-specific DNA cleavage by the Cas9-gRNA complex. Nucleofection achieved up to $70 \%$ transfection efficiency and showed consistency across different organoid lines (Figure 1-figure supplement 1a and b). To optimise site-specific DNA cleavage, we 
used nucleofection to introduce the Cas9-gRNA complex into cells in different forms (Figure 1-figure supplement 1c). Consistent with previous reports, Cas9 ribonucleoproteins (RNPs) outperformed plasmid-based Cas9 approaches (Kim et al., 2014; Lin et al., 2014), both in the T7 endonuclease assay and an online CRISPR editing analysis tool (Figure 1-figure supplement 1d). Thus, we adopted nucleofection and single-stranded synthetic gRNA combined with an RNP (ssRNP) for downstream experiments. This strategy has the advantage that the RNP is rapidly degraded and should produce minimal off-target effects.

To establish our gene targeting workflow, we first focused on generating a $\beta$-actin (ACTB)-fusion protein, taking advantage of the abundance of ACTB protein in human foetal lung organoids and a previously published targeting strategy (Roberts et al., 2017). We designed a repair template to generate an $\mathrm{N}$ terminal monomeric (m)EGFP-ACTB fusion based on the most efficient gRNA tested (Figure 1a). We set the following rules for repair template design to facilitate efficient and consistent gene targeting: (1) protospacer adjacent motif (PAM) sequence mutated to prevent editing by ssRNP (Paquet et al., 2016); (2) 700-1000 nt length of each homologous arm (Yao et al., 2018); (3) minimal plasmid size to maximise delivery into organoid cells ( $<7.0 \mathrm{~kb}$ in size recommended empirically) and (4) monomeric forms of fluorescent protein to avoid undesirable fusion protein aggregates. As expected, $72 \mathrm{hr}$ after nucleofection of the ssRNP and repair template, mEGFP ${ }^{+}$organoid cells could be enriched by flow cytometry (Figure $1 \mathrm{~b}$ and $\mathrm{c}$ ). These cells were collected and pooled together, but seeded sparsely, and successfully expanded into organoid colonies (Figure 1d). The mEGFP-ACTB fusion protein localised to cell-cell junctions as expected (Roberts et al., 2017). These small colonies could be further expanded into new organoid lines and $59 \%$ of lines ( $n=17 / 29$ lines, from $N=2$ parental organoid lines, Supplementary file 1) were correctly targeted. Targeted organoids continued to express the multipotent lung progenitor marker, SOX9 (Figure 1e). We sought to further increase targeting efficiency using drugs previously reported to enhance HDR (Maruyama et al., 2015; Song et al., 2016; Yu et al., 2015). However, using flow cytometry as a simple assay, none of the drugs tested increased the rate of gene targeting (Figure 1-figure supplement 2).

The AAVS1 locus (Adeno-Associated Virus Integration Site 1, located in the first intron of the PPP1R12C gene) has been considered to be a 'safe harbour locus' for expressing exogenous genes in a controllable manner in human cells without silencing (Smith et al., 2008). As a further proof of concept, we successfully targeted AAVS1 to express a membrane tagged TagRFP-T (mTagRFP-T) for cell shape visualisation (Figure $1 \mathrm{f}$ and $g ; n=4 / 6$ correctly targeted lines from $N=1$ parental organoid line). Therefore, we have combined Cas9 RNP with single-stranded synthetic gRNA, a simple circular plasmid repair template and a strategy to enrich correctly targeted cells via flow cytometry for gene targeting in human foetal lung organoids. We named this workflow Organoid Easytag.

To expand our Organoid Easytag pipeline to reporter generation, we targeted SOX9. SOX9, a transcription factor, is a tip progenitor cell marker for developing lungs (Nikolić et al., 2017), and SOX9 reporters are useful for monitoring organoid progenitor state. To overcome its low expression level, we used a Histone H2B-EGFP fusion (H2B-EGFP hereafter) to concentrate the EGFP signal in the nucleus (Figure 2a). A T2A sequence, a self-cleavage peptide, was also inserted between SOX9 and $H 2 B-E G F P$ to ensure that SOX9 protein was minimally influenced. This strategy allowed us to enrich correctly targeted cells by flow cytometry. Targeted colonies could be expanded and maintained normal SOX2, SOX9 and NKX2-1 expression (Figure 2b, Figure 2-figure supplement 1a; n = 9/23 correctly targeted lines from $\mathrm{N}=3$ parental organoid lines). Importantly, we noted that although we were only able to generate SOX9 reporter lines as heterozygotes (Figure 2-figure supplement $1 \mathrm{~b}$ and $c$ ), the gRNA sites in the wildtype alleles were intact (6/6 lines tested, $N=3$ parental organoid lines) (Figure 2-figure supplement 1d). This offers the opportunity to retarget the second allele if desired. To evaluate potential off-target effects, we selected the top nine most probable SOX9 gRNA off-target sites (Figure 2-figure supplement 1e) and tested if these regions contained any undesired CRISPR-induced scars. No indels were detected within 200 bps flanking these potential off-target sites (Figure 2-figure supplement 1e and f), suggesting low off-target effects of our workflow (3/3 SOX9-T2A-H2B-EGFP lines tested). Additionally, we validated that the SOX9-targeted organoids retained the potential to differentiate (Figure 2-figure supplement $1 \mathrm{~g}$ ), further demonstrating the normality of the targeted organoid lines.

We sought to apply Organoid Easytag to transcriptionally silent loci to generate differentiation reporters. We adopted the strategy of inserting an exogenous promoter flanked by two Rox sites 
a Nucleofection
EGFP+ cells sorted

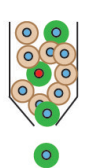

Individual colony picked
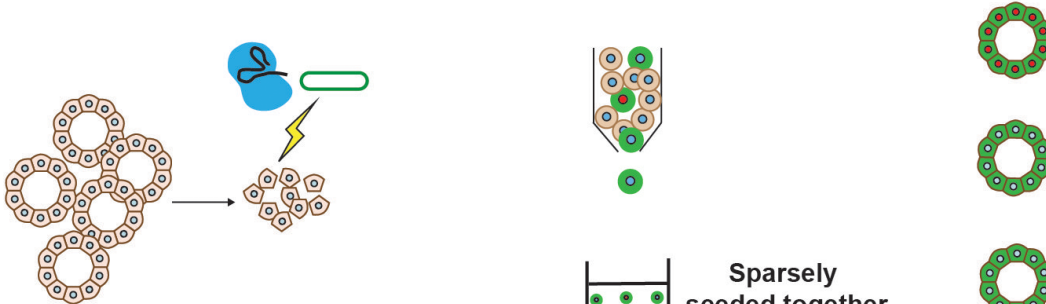

Day 0

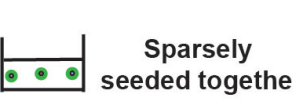

Day 3

\section{C}
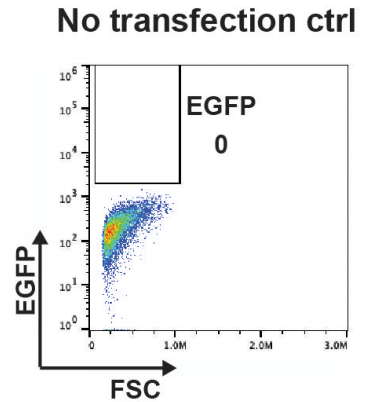

Genotyping

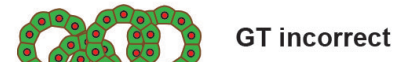

b ACTB gene targeting design

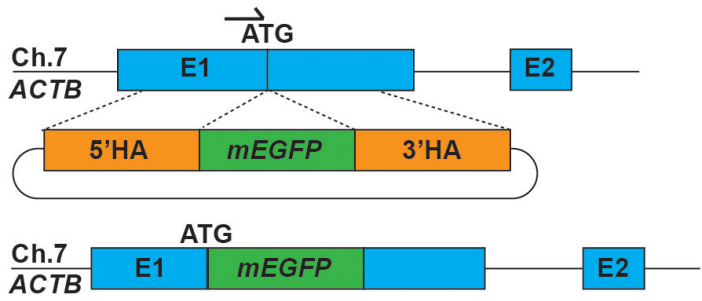

\section{d mEGFP-ACTB organoid}
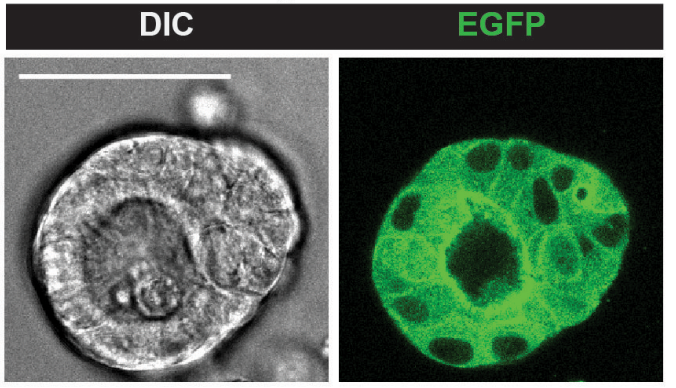

\section{f AAVS1 gene targeting design}

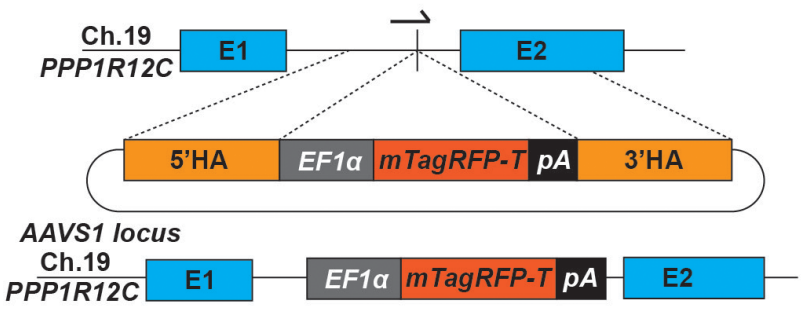

\section{e $m E G F P-A C T B$}

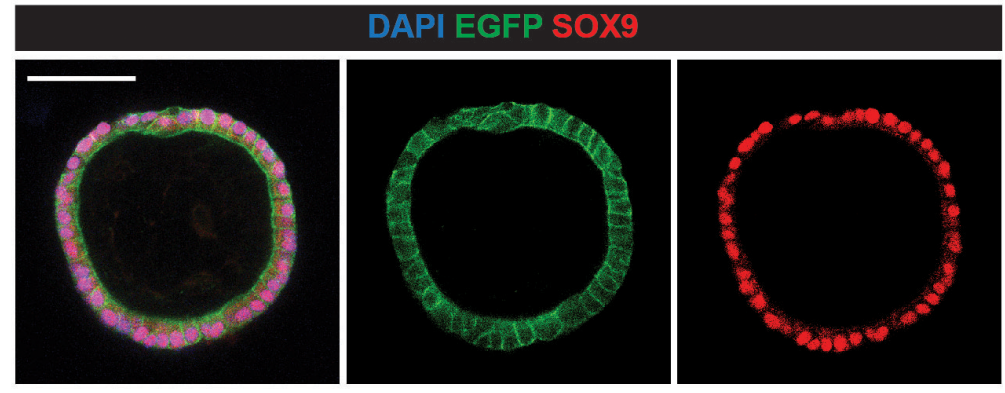

9 AAVS1-EF1a-mTagRFP-T

\section{DAPI IITagRFP-T SOX2}
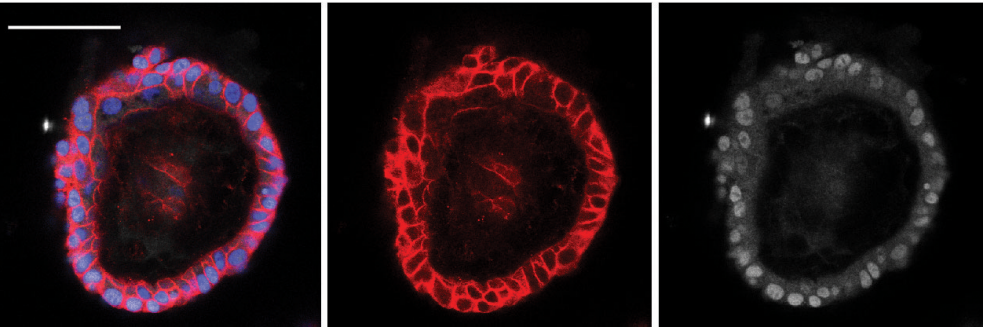

Figure 1. The Organoid Easytag workflow for gene targeting in human organoids. (a) Schematic of the Organoid Easytag workflow. ssRNP and a circular plasmid repair template are nucleofected into dissociated cells at day 0 . By day 3, cells have proliferated to become tiny colonies and are removed from the Matrigel and dissociated for selection by flow cytometry. EGFP+ cells are re-plated sparsely ( 1000-1500 cells/well of a 24-well plate) and grown until day 15 when organoids reach a sufficient size to be manually picked under a fluorescent microscope. Typically, 10-40 organoid colonies formed per 1000 cells seeded. Organoids are picked into individual wells and passaged until sufficient cells are obtained for both genotyping and freezing down the line. Cells with red nuclei represent incorrectly targeted cells. Cells with white nuclei denote correctly targeted cells. (b) Schematic of repair template design for $\mathrm{N}$ terminal fusion mEGFP-ACTB gene targeting and the final product. Arrow shows the position of gRNA. E1, exon 1; E2, exon 2; 5'HA, 5' homology arm; 3'HA, 3' homology arm. (c) Representative flow cytometry results showing the percentage of EGFP cells 72 hr after nucleofection is performed. (d) Representative image showing mEGFP-ACTB organoid. DIC channel on the left and EGFP channel on the right. (e) Immunofluorescence of mEGFP-ACTB organoids. Blue: DAPI (nuclei); green: EGFP (ACTB fusion protein); red: SOX9 (lung progenitor marker).

Figure 1 continued on next page 
Figure 1 continued

(f) Schematic of the AAVS1 targeting repair template design and the final product. E1, exon 1, E2, exon 2. Arrow indicates the position of the gRNA. (g) Immunofluorescence of AAVS1-EF1a-mTagRFP-T organoids. Blue: DAPI (nuclei); red: mTagRFP-T (membrane localised reporter); white: SOX2 (lung progenitor marker). Scale bars in all panels denote $50 \mu \mathrm{m}$.

The online version of this article includes the following figure supplement(s) for figure 1:

Figure supplement 1. Optimisation of DNA delivery and CRISPR cutting.

Figure supplement 2. Small molecules did not significantly improve organoid gene targeting efficiency.

which could be subsequently excised by Dre recombinase (Anastassiadis et al., 2009; Roberts et al., 2019). The exogenous EF1a promoter drives fluorescent reporter expression for flow cytometry selection, allowing us to first positively enrich correctly targeted cells $\left(V_{e n u s}^{+}\right.$), and subsequently enrich (Venus') cells following Dre-mediated EF1a removal (Figure 2-figure supplement 2a). This design also helps to minimise the repair template size. We targeted the SFTPC (surfactant protein C, Figure 2-figure supplements 2-3) and TP63 genes (Figure 2-figure supplement 4) because they are not expressed in human foetal lung organoid cells and are well-established markers for alveolar type II and basal cell lineages, respectively (Barkauskas et al., 2013; Rock et al., 2009). To test SFTPC reporter function, we overexpressed NKX2-1 from a lentiviral vector (Figure 2-figure supplement 2d; Lim et al., 2021). Following NKX2-1 induction, Venus was co-expressed with proSFTPC protein, confirming that the reporter is functional (Figure 2-figure supplement 2e). We noted that the Venus signal was cytoplasmic, likely due to inefficient self-cleavage of the T2A sequence (Figure 2-figure supplement 2f). TP63 reporter organoid lines were produced using the same strategy (Figure 2figure supplement 4). These results indicate that the Organoid Easytag workflow can target silent gene loci.

The generation of straightforward gene KOs by induction of indels using the CRISPR-Cas9 system can suffer from translation retention and exon skipping (Smits et al., 2019; Tuladhar et al., 2019). Moreover, in the absence of a strong, immediate phenotype the KO cells cannot readily be identified. We sought to solve these problems by generating a gene $\mathrm{KO}$ in a controlled manner using Organoid Easytag. We focused on SOX2 as its function remains to be elucidated in human foetal lung progenitors. We replaced the SOX2 coding sequence (CDS) with T2A-H2B-EGFP to generate SOX2 $\mathrm{KO}$ organoids. Using two gRNAs targeting the $\mathrm{N}$ and $\mathrm{C}$ terminal of the SOX2 CDS, respectively, we sequentially replaced both copies of the SOX2 CDS (Figure 2c, Figure 2-figure supplement 5). The EGFP signal was increased after targeting the second copy of the SOX2 CDS, making selection of serially targeted alleles by flow cytometry straightforward (Figure 2-figure supplement 5c). SOX2 $\mathrm{KO}$ colonies proliferate and grow normally for at least four serial passages, suggesting that SOX2 is not crucial for human foetal lung tip progenitor cell self-renewal (Figure 2d).

Additionally, we tested the Organoid Easytag workflow in the human foetal intestinal organoid system (Elmentaite et al., 2020) and successfully derived SOX9 reporter lines (Figure $2 e$ and f; 2/4 lines tested correctly targeted).

We have demonstrated that our Organoid Easytag pipeline can target various loci, including highly abundant genes, transcription factors, the human safe harbour locus and transcriptionally silent genes. The Organoid Easytag workflow can also be adapted to generate KOs and applied to different organoid systems (efficiency details summarised in Supplementary file 1). Organoid Easytag will be a complementary technique to the recently published CRISPR-HOT approach (Artegiani et al., 2020) and is particularly suited for experiments where precise gene editing is required (Figure 2-figure supplement $5 f$ ).

KOs are not suitable for studying the function of genes which are required for stem cell selfrenewal. Moreover, temporal, and reversible, control of gene expression cannot be easily achieved using KOs. Inducible CRISPRi could potentially solve these problems. We first embedded an N-terminal KRAB-dCas9 fusion (Mandegar et al., 2016) in a doxycycline (Dox)-inducible TetON system aiming to gain temporal control of CRISPRi function. However, the TetON system alone did not provide sufficiently tight control of CRISPRi, possibly due to copy number or integration position of the transgene (Figure 3-figure supplement 1). To solve this problem, we fused KRAB-dCas9 with a destabilising domain derived from Escherichia coli dihydrofolate reductase (DHFR) (Figure 3a). DHFR is stabilised by a small molecule, trimethoprim (TMP) (Figure 3b; Iwamoto et al., 2010). We evaluated the new 


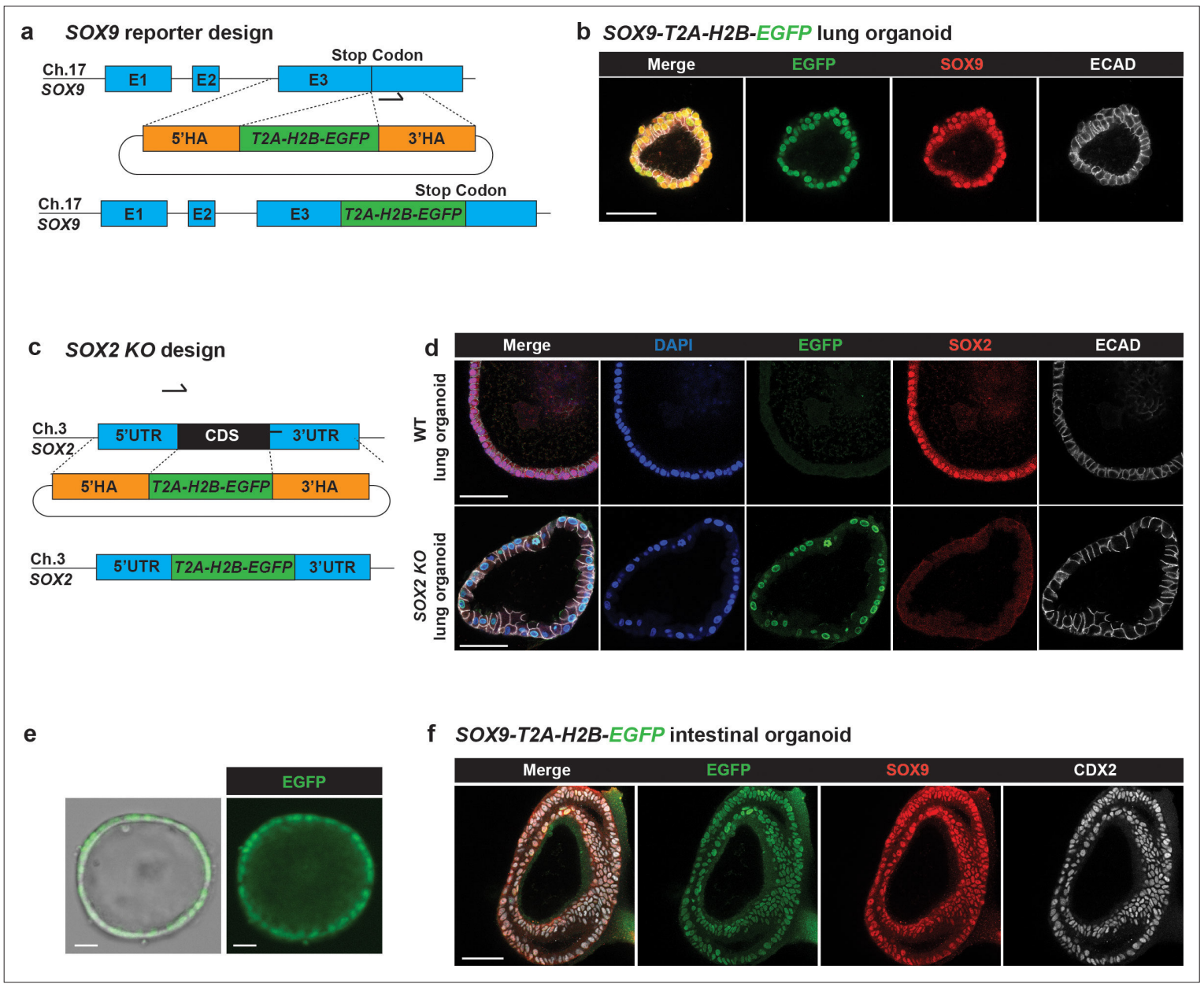

Figure 2. The Organoid Easytag workflow can be adapted for multiple applications. (a) Schematic of the SOX9 locus repair template design and final product. E1, exon 1; E2, exon 2; E3, exon 3; 5' HA, 5' homology arm; 3' HA, 3' homology arm. Arrow shows the position of the gRNA. (b) Immunofluorescence of SOX9-T2A-H2B-EGFP human foetal lung organoids. Green: EGFP (SOX9 reporter); red: SOX9 (lung progenitor marker); white: ECAD (E-cadherin, basal-lateral junctions). (c) Schematic showing repair template design and final product for the generation of SOX2 knockout organoids using Organoid Easytag. Two gRNA sites were used at the $\mathrm{N}$ and $\mathrm{C}$ terminal of the SOX2 CDS, respectively. SOX2 CDS was replaced by T2A-H2B-EGFP. (d) Representative immunofluorescence showing that SOX2 protein is completely knocked out. Blue: DAPI (nuclei); green: EGFP (SOX2 transcriptional reporter); red: SOX2 protein (lung progenitor marker); white: ECAD (E-cadherin, basal-lateral junctions). (e) Widefield microscopic images showing SOX9 reporter human foetal intestinal organoid. (f) Immunofluorescence of SOX9-T2A-H2B-EGFP human foetal intestinal lung organoids. Green: EGFP (SOX9 reporter); red: SOX9; white: CDX2 (intestinal lineage marker). Scale bars denote $50 \mu \mathrm{m}$.

The online version of this article includes the following figure supplement(s) for figure 2 :

Figure supplement 1. Characterisation of SOX9 targeted colonies.

Figure supplement 2. Organoid Easytag to target silent gene, SFTPC locus.

Figure supplement 3. Characterisation of SFTPC reporter organoids.

Figure supplement 4. Organoid Easytag to target the TP63 locus.

Figure supplement 5. Generation of SOX2 knockout using Organoid Easytag workflow.

inducible CRISPRi system by depleting a ubiquitous cell surface marker CD71 (transferrin receptor C [TFRC]). Using previously validated gRNAs (Horlbeck et al., 2016), CD71 protein was depleted in the majority of the cells after 5 days of Dox and TMP treatment $(91.4 \% \pm 2.1 \%$, mean \pm SEM, $N=3$; Figure $3 c$ and d; Figure 3-figure supplement $2 a$ and b). Whereas no knockdown was observed in the no Dox/TMP treatment group, or in organoids transduced with non-targeting control gRNA. The knockdown effect was achieved after 5 days, could be reversed after 1-2 weeks of Dox and TMP removal and then induced again (Figure 3-figure supplement 2a-c), consistent with a recent report (Nuñez et al., 2021). We further evaluated the inducible CRISPRi system using SOX2. SOX2 
a

CRISPRi:

\begin{tabular}{l|l|l|} 
pLenti & TetON & KRAB-dCas9 \\
\hline
\end{tabular}

gRNA:

pLenti U6 gRNA EF1a EGFP-CAAX b

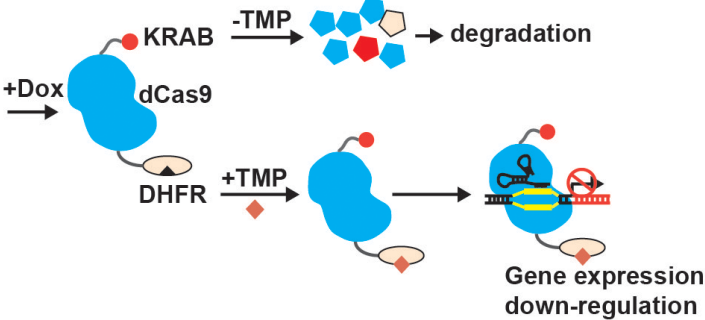

C

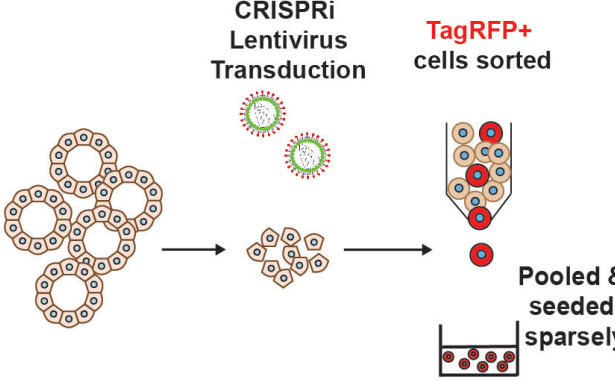

e

d

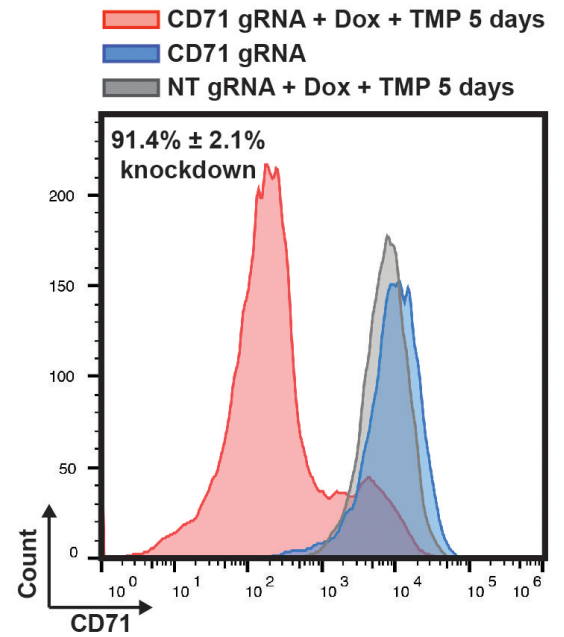

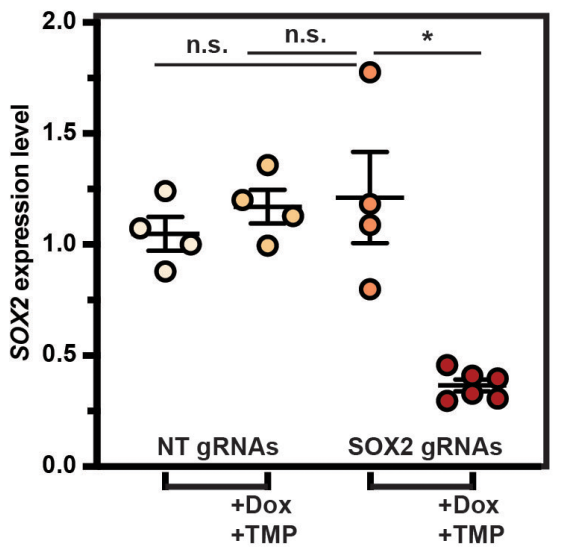

f

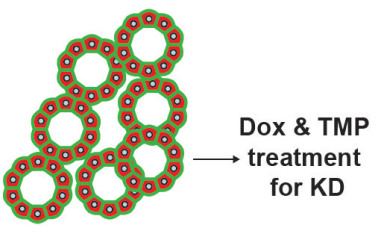

atment

Day 33

\section{\%\%}
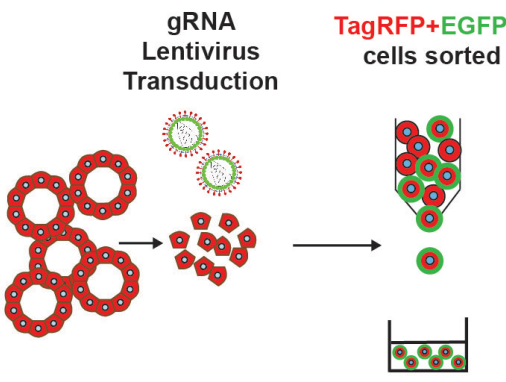

Day 23

\section{NT gRNA control}

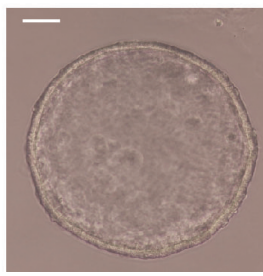

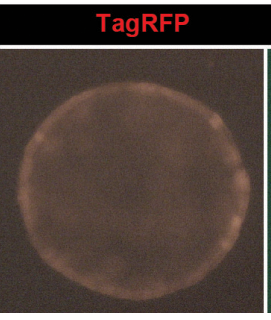

h SOX2 CRISPRi

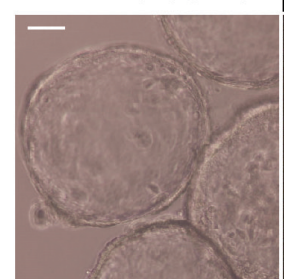

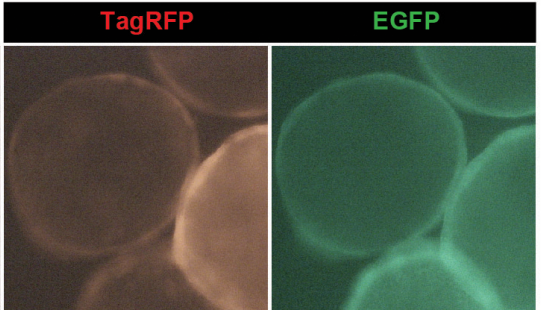

Figure 3. Precise temporal control of CRISPRi in human foetal lung organoids can be achieved via combining the doxycycline (Dox)-inducible system with a protein destabilising domain (DD). (a) Schematic of lentiviral vector designs for sequential introduction of inducible CRISPRi and gRNAs into human foetal lung organoids. N-terminal KRAB-dCas9 was fused with dihydrofolate reductase (DHFR)-derived DD for stringent control of CRISPRi function. EGFP was fused with a C-terminal CAAX domain to achieve membrane labelling of the transduced cells. (b) Schematic showing how the temporal control of CRISPRi protein is achieved by combining the Dox-inducible system and DD. The Dox-inducible system is somewhat leaky, but in the absence of trimethoprim (TMP), any KRAB-dCas9-DHFR fusion protein produced is degraded. Dox treatment results in high levels of KRAB-dCas9DHFR fusion protein expression. When TMP is not present, the fusion protein is destabilised, whereas when TMP is supplemented, the fusion protein

Figure 3 continued on next page 
Figure 3 continued

is stabilised and can exert its gene expression repression function. (c) Workflow for generating gene knockdown (KD) using CRISPRi system. A parental organoid line with inducible CRISPRi was produced via lentiviral transduction followed by sorting for TagRFP-positive cells. Single cells were re-plated ( 3000-5000 cells/well of a 24-well plate) and expanded for around 17 days. gRNA lentivirus was then introduced by a second lentiviral transduction event, followed by sorting for TagRFP/EGFP dual-positive cells after 3 days. Cells were re-plated ( 2000-3000 cells/well of a 24-well plate) and expanded for another 10 days before treatment with Dox and TMP. 4-6 days after Dox and TMP treatment, gRNA performance was evaluated. (d) Representative flow cytometry results for validation of inducible CRISPRi performance using CD71 as a target. After TMP and Dox administration for 5 days, CD71 protein was successfully downregulated. Grey histogram indicates CD71 expression level for organoids with non-targeting (NT) control gRNA after Dox and TMP treatment for 5 days; blue histogram indicates the CD71 expression level for organoids with CD71 gRNA without Dox or TMP treatment, showing no KD effect; pink histogram indicates the CD71 expression level for CD71 gRNA with Dox and TMP treatment for 5 days. The figures on the graph indicate the percentage of CD71 low cells, mean \pm SEM. Two independent organoid lines with two different CD71 gRNAs were used. (e) SOX2 can be effectively knocked down using inducible CRISPRi without leaky effects indicated by qRT-PCR. Organoids with Dox and TMP treatment were harvested 5 days after the treatment. Each dot represents an independent experiment. Two independent organoid lines with two different NT control gRNAs and three different SOX2 gRNAs were used. SOX2 expression level was normalised to organoids with NT control gRNAs. Detailed breakdown of these data is shown in Figure 4-figure supplement 1. Error bars are plotted to show mean \pm SEM. A two-sided Student's t-test with non-equal variance was used to compare the means of each group. * indicates $\mathrm{p}$-value $<0.05$. n.s. indicates non-significant. (f) Representative western blot results showing SOX2 was effectively knocked down using the inducible CRISPRi system (gRNA7 and gRNA8 are independent guides targeted to SOX2; N = 2 parental organoid lines used). (g-, h) Representative images showing organoid morphology after SOX2 knockdown. Images were taken after 5 days of Dox and TMP treatment. SOX2 knockdown (right panel) did not result in obvious phenotypic changes in organoids compared with NT gRNA control (left panel). Scale bars denote $100 \mu \mathrm{m}$.

The online version of this article includes the following figure supplement(s) for figure 3 :

Figure supplement 1. The tetON system alone was not able to tightly control CRISPRi function.

Figure supplement 2. Kinetics and reversibility of the inducible CRISPRi system.

could be efficiently knocked down at both the transcriptional and protein level and no background CRISPRi function was observed (Figure $3 e$ and f). The SOX2 knockdown occurred by day 1 and was also reversible (Figure 3-figure supplement 2d), indicating that the CRISPRi function is efficient and tightly regulated. Consistent with our SOX2 KO, we did not observe effects on organoid morphology or growth after SOX2 knockdown (Figure $3 g$ and $h$ ). This further confirmed our finding that SOX2 is not crucial for organoid self-renewal.

Finally, we have assembled the CRISPRa system to switch on endogenous genes in human foetal lung organoids. We tested a previously reported CRISPRa system, dxCas9(3.7)-VPR (Hu et al., 2018), for its ability to activate SFTPC transcription (Figure 4). Inducible CRISPRa can induce SFTPC gene expression efficiently at both transcriptional and protein level without influencing organoid growth (Figure 4b-d).

We have established a complete organoid genetic toolbox for gene targeting, reporter generation, controllable gene KOs, inducible gene knockdown and gene activation in the human foetal lung organoid system (efficiency details provided in Supplementary file 1). We envision that these tools can be easily adapted for organoid systems derived from other tissues that are suitable to be nucleofected, or transduced by lentivirus. This will empower tissue-derived organoids to model human congenital lung disease and benchmark gene function using the Human Cell Atlas as a reference.

\section{Materials and methods Human embryonic and foetal lung tissue}

Human embryonic and foetal lungs were obtained from terminations of pregnancy from Cambridge University Hospitals NHS Foundation Trust under permission from the NHS Research Ethical Committee (96/085) and the Joint MRC/Wellcome Trust Human Developmental Biology Resource (London and Newcastle, http://www.hdbr.org/) under permission from the NHS Research Ethics Committee (18/ LO/0822 and 18/NE/0290). Samples used had no known genetic abnormalities.

\section{Derivation and maintenance of human foetal lung organoid culture}

Human foetal lung organoids were derived and maintained as previously reported (Nikolić et al., 2017). Briefly, human foetal lung tissues were dissociated using Dispase $(8 \mathrm{U} / \mathrm{ml}$, Thermo Fisher Scientific, 17105041) at room temperature (RT) for $2 \mathrm{~min}$. Mesenchyme was dissected away using needles. Tips of the branching epithelium were micro-dissected, transferred into $50 \mu \mathrm{l}$ of Matrigel (356231, 
a

CRISPRi:

pLenti TetON dxCas9(3.7)-VPR EF1a TagRFP-2A-tet3G-

gRNA:

pLenti U6 gRNA EF1a EGFP-CAAX

b

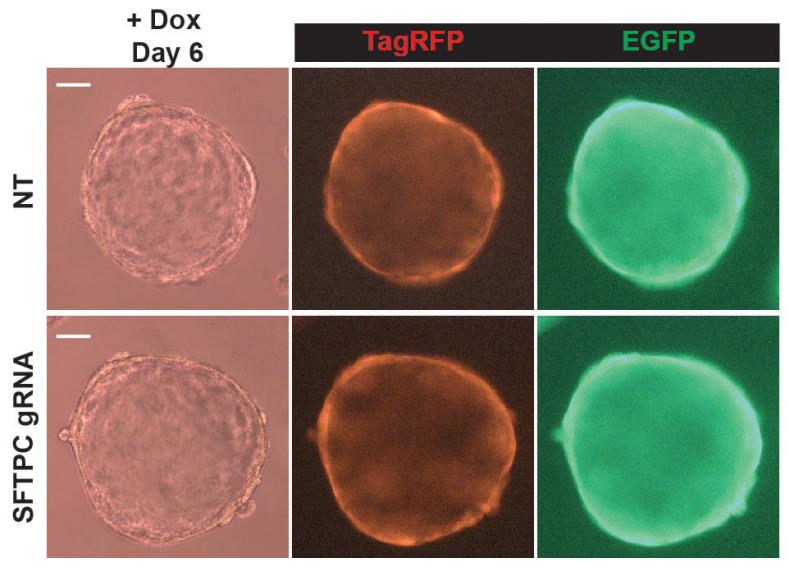

C

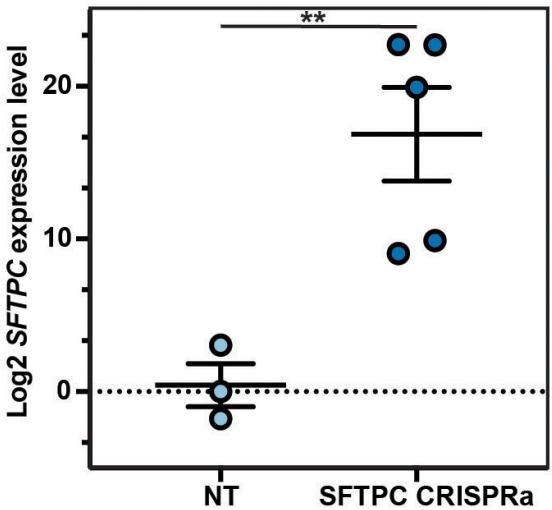

d

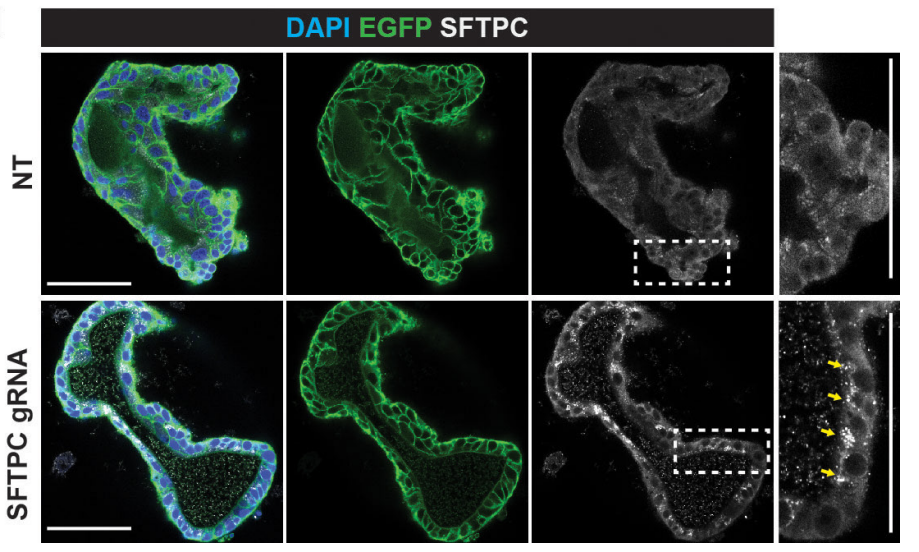

Figure 4. Inducible CRISPRa drove expression of a silent gene, SFTPC, in human foetal lung organoids. (a) Schematic of lentiviral design for introducing the CRISPRa system and gRNA into human foetal lung organoids. (b) Representative images to show organoid morphology after SFTPC activation after 6 days of doxycycline (Dox) treatment. SFTPC activation (lower panel) did not result in obvious phenotypic changes in organoids compared with non-targeting gRNA control (upper panel). (c) SFTPC can be effectively activated by CRISPRa system indicated by qRT-PCR. Each dot represents an independent experiment. Two independent organoid lines with two different non-targeting control (NT) gRNAs and three different SFTPC gRNAs were used. SFTPC expression level was normalised to organoids with non-targeting control (NT) gRNAs. A detailed breakdown of the results is given in Figure 4-figure supplement 1. Error bars are plotted to show mean \pm SEM. A two-sided Student's t-test with non-equal variance was used to compare the means of each group. ${ }^{*}$ indicates $p$-value $<0.01$. (d) Representative immunofluorescence showing proSFTPC protein accumulation in organoid cells after CRISPRa activation of SFTPC gene expression. Bright SFTPC protein aggregates accumulated in cells, consistent with the hydrophobic nature of SFTPC protein. Green: EGFP; white: proSFTPC protein. Scale bars denote $100 \mu \mathrm{m}$ (b) and $50 \mu \mathrm{m}$ (d).

The online version of this article includes the following figure supplement(s) for figure 4:

Figure supplement 1. SOX2 CRISPRi and SFTPC CRISPRa results break down.

Corning) and seeded in one well of a 24-well low-attachment plate (M9312-100EA, Greiner). The plate was incubated at $37^{\circ} \mathrm{C}$ for 5 min to solidify the Matrigel. $600 \mu$ of self-renewing medium containing N2 (1:100, Thermo Fisher Scientific, 17502-048), B27 (1:50, Thermo Fisher Scientific, 12587-010), $\mathrm{N}$-acetylcysteine (1.25 mM, Merck, A9165), EGF (50 ng/ml, PeproTech, AF-100-15), FGF10 (100 ng/ ml, PeproTech, 100-26), FGF7 (100 ng/ml, PeproTech, 100-19), Noggin (100 ng/ml, PeproTech, $120-$ 10C ), R-spondin (5\%, v/v, Stem Cell Institute, University of Cambridge), CHIR99021 (3 $\mu$ M, Stem Cell Institute, University of Cambridge) and SB 431542 (10 $\mu \mathrm{M}$, Bio-Techne, 1614) was added. The plate was incubated under standard tissue culture conditions $\left(37^{\circ} \mathrm{C}, 5 \% \mathrm{CO}_{2}\right)$. Once formed, organoids were maintained in self-renewing medium and passaged by mechanically breaking using P200 pipettes every 10-14 days.

\section{Whole-mount immunostaining for human foetal lung organoids}

Organoids were fixed with 4\% paraformaldehyde (PFA) directly in the culture plates on ice for 30 min. After two PBS washes, $0.5 \%$ (w/v) bovine serum albumin (BSA), $0.2 \%$ Triton-X in PBS (washing solution) was added and left on ice overnight to dissolve Matrigel. Organoids were then transferred into 
multiple CellCarrier-96 Ultra Microplates (PerkinElmer, 6055300) for staining. Subsequently, blocking was performed in 5\% donkey serum (Stratech, 017-000-121-JIR), 0.5\% (w/v) BSA, 0.2\% Triton-X in PBS (blocking solution) at $4^{\circ} \mathrm{C}$ overnight. For primary antibody staining, the following antibodies in blocking solution were used at $4^{\circ} \mathrm{C}$ overnight: SOX2 (1:500, Bio-Techne, AF2018, RRID:AB_355110), SOX9 (1:500, Merck, AB5535, RRID:AB_2239761), E-cadherin (1:1500, Thermo Fisher Scientific, 13-1900, RRID:AB_2533005), NKX2-1 (1:500, Abcam, ab76013, RRID:AB_1310784), TagRFP (1:1000, Evrogen, AB233, RRID:AB_2571743), GFP/Venus (1:500, Abcam, ab13970, RRID:AB_300798), CDX2 (1:200, Abcam, ab157524, RRID:AB_2721036) and proSFTPC (1:500, Merck, AB3786, RRID:AB_91588). After washing off the primary antibodies, the following secondary antibodies in washing buffer were used at $4^{\circ} \mathrm{C}$ overnight: donkey anti-chick Alexa 488 (1:2000, Jackson Immune, 703-545-155, RRID:AB_2340375), donkey anti-rabbit Alexa 594 (1:2000, Thermo Fisher Scientific, A-21207, RRID:AB_141637), donkey anti-goat Alexa 594 (1:2000, Thermo Fisher Scientific, A-11058, RRID:AB_2534105), donkey antigoat Alexa 647 (1:2000, Thermo Fisher Scientific, A-21447, RRID:AB_2535864), donkey anti-rat Alexa 647 (1:2000, Jackson Immune, 712-605-153, RRID:AB_2340694) and donkey anti-mouse Alexa 647 (1:2000, Thermo Fisher Scientific, A31571, RRID:AB_162542). The following day, DAPI (Sigma, D9542) staining was performed in washing solution at $4^{\circ} \mathrm{C}$ for $30 \mathrm{~min}$. After two washes with PBS, 97\% (v/v) 2'-2'-thio-diethanol (TDE, Merck, 166782) in PBS was used for mounting. Confocal z stacks were acquired using Leica SP8 at an optical resolution of $1024 \times 1024$ using a $\times 40$ lens. Single-plane images are shown. Images were processed using ImageJ (version 2.0.0).

\section{Plasmid nucleofection}

For testing transduction efficiency, organoids were dissociated into single cells using pre-warmed TrypLE Express (12605028, Thermo Fisher Scientific) at $37^{\circ} \mathrm{C}$ for $10 \mathrm{~min}$. The reaction was terminated by adding Advanced DMEM/F12 (12634028, Thermo Fisher Scientific) and cells passed through a $30 \mu \mathrm{m}$ cell strainer. $2 \times 10^{5}$ organoid single cells were re-suspended with Lonza P3 nucleofection buffer and $1 \mu$ l of pmaxGFP (Lonza) and transferred to $20 \mu$ l nucleofection cuvette (V4XP-3024, Lonza). Nucleofection was performed using Lonza 4D Nucleofector with $X$ unit using the program EA125. After nucleofection, self-renewing medium supplemented with $10 \mu \mathrm{M}$ Y-27632 (ROCK inhibitor, ROCKi, 688000, Merck) was added to dilute the P3 buffer. Cell mixture was then seeded in Matrigel in two wells of a 24-well plate and cultured with self-renewing medium with ROCKi (10 $\mu \mathrm{M})$ for $72 \mathrm{hr}$ before FACS analysis.

\section{Lentiviral production}

HEK293T cells were kindly provided by Dr. Rick Livesey and tested mycoplasma negative. We grew HEK293T cells in $10 \mathrm{~cm}$ dishes to a confluence of $80 \%$ before we transfected the lentiviral vector $(10 \mu \mathrm{g})$ with packaging vectors including pMD2.G (3 $\mu \mathrm{g}$, Addgene plasmid \# 12259), psPAX2 (6 $\mu$ g, Addgene plasmid \# 12260) and pAdVAntage (3 $\mu$ g, E1711, Promega) using Lipofectamine 2000 Transfection Reagent (11668019, Thermo Fisher Scientific) according to the manufacturer's protocol. After $16 \mathrm{hr}$, medium was refreshed. Supernatant containing lentivirus was harvested at $24 \mathrm{hr}$ and $48 \mathrm{hr}$ after medium refreshing and pooled together. Supernatant was centrifuged at $300 \mathrm{~g}$ to remove cell fragments and passed through $0.45 \mu \mathrm{m}$ filter. Lentivirus containing $>10 \mathrm{~kb}$ length insert (inducible CRISPRi and CRISPRa) was concentrated using AVANTI J-30I centrifuge (Beckman Coulter) with JS-24.38 swing rotor at $72,000 \mathrm{~g}$ for $2 \mathrm{hr}$ at $4^{\circ} \mathrm{C}$ and pellets were dissolved in $200 \mu \mathrm{l}$ PBS. Other lentivirus, including gRNA and NKX2-1-inducible overexpression, were concentrated using Lenti-X Concentrator (631232, Takara) and pellets were dissolved in $400 \mu$ PBS.

\section{Lentivirus infection of organoids to test infection efficiency}

Organoid single-cell suspension was prepared as for nucleofection. $5 \mu$ lentivirus (CMV-myrAKTIRES-GFP) suspension was applied to $2 \times 10^{5}$ organoid single cells suspended in $500 \mu$ l self-renewing medium with $10 \mu \mathrm{M} \mathrm{ROCKi}$ (without Matrigel) in one well of 24 -well plate and incubated at $37^{\circ} \mathrm{C}$ overnight. The following day, cells were harvested and washed twice with PBS before pelleting and seeding in Matrigel in two wells of a 24-well plate. Cells were grown in self-renewing medium with ROCKi $(10 \mu \mathrm{M})$ for $72 \mathrm{hr}$ before flow cytometry. CMV-myrAKT-IRES-GFP was only used for checking lentiviral transduction efficiency (Supplementary file 1). 


\section{Lipofectamine transfection of organoids}

Organoid single cells were prepared the same way as for nucleofection. For comparing transduction efficiency, $1 \mu \mathrm{g}$ of pmaxGFP (Lonza) was mixed with $1 \mu$ l of Lipofectamine Stem Transfection Reagent (STEM00001, Thermo Fisher Scientific) according to the manufacturer's protocol. $50 \mu \mathrm{l}$ reaction mixture was applied to $2 \times 10^{5}$ organoid single cells suspended with $450 \mu$ self-renewing medium with ROCKi (without Matrigel) in a single well of a 24 -well plate. The plate was then centrifuged at $32^{\circ} \mathrm{C}$ at $600 \mathrm{~g}$ for $1 \mathrm{hr}$, followed by incubation at $37^{\circ} \mathrm{C}$ for $2-4 \mathrm{hr}$. Cells were then harvested, pelleted and seeded in Matrigel in two wells of a 24-well plate and grown in self-renewing medium supplemented with ROCKi $(10 \mu \mathrm{M})$ for $72 \mathrm{hr}$ before FACS analysis.

\section{Nucleofection for gene targeting in human foetal lung organoids}

Cas9 protein was prepared and used as previously reported (Bruntraeger et al., 1961). If synthetic single-strand gRNAs were used, $2 \mu \mathrm{l}$ spCas9 $(4 \mu \mathrm{g} / \mu \mathrm{l})$ and $2.88 \mu \mathrm{l}$ of single-strand synthetic RNA (ssRNA, $100 \mu \mathrm{M}$, Synthego) were mixed and incubated at RT for a minimum of $10 \mathrm{~min}$ in order to form ssRNPs. If synthetic crispr/tracer (cr/tr) RNA heterodimers were used, 200 pmol synthetic cr RNA (IDT) and 200 pmol synthetic tr RNA (IDT) were mixed with $2.5 \mu$ l Nuclease Free Duplex Buffer (11-01-03-01, IDT) and denatured at $95^{\circ} \mathrm{C}$ for $2 \mathrm{~min} .2 \mu \mathrm{lof} \mathrm{cr} / \mathrm{tr}$ RNA heterodimer was cooled down to RT on the bench, mixed with $2 \mu \mathrm{lspCas} 9(4 \mu \mathrm{g} / \mu \mathrm{l})$ and incubated at RT for a minimum of $10 \mathrm{~min}$ to form cr/tr RNPs. At the same time, organoids were dissociated into single cells, according to the protocol previously described for nucleofection. $4 \times 10^{5}$ cells were suspended using Lonza Nucleofection P3 buffer, mixed with $10 \mu \mathrm{g}$ of appropriate plasmid repair template. The cell suspension was further mixed with pre-formed Cas9 RNPs and equally distributed into two $20 \mu$ cuvettes (V4XP-3032, Lonza). Nucleofection was performed using program EA104 for human foetal lung organoids (EA125 could also be used, but resulted in more cell death). After nucleofection, self-renewing medium with ROCKi was added to dilute the P3 buffer. The cell mixture was then taken out and seeded in Matrigel in four wells of a 24-well plate and cultured with self-renewing medium with $10 \mu \mathrm{M}$ ROCKi for 72 hrs before flow cytometry.

All gRNAs used for gene targeting were pre-tested for efficient DNA cleavage activity using the T7 endonuclease assay and ICE analysis (at least 10\% indels, typically 20-30\%) before repair template design. Sequences used:

\begin{abstract}
ACTB 5'-GCTATTCTCGCAGCTCACCA TGG, SOX9 5'-CTTGAGGAGGCCTCCCACGA AGG, AAVS1 5'-GTCCCCTCCACCCCACAGTG GGG, SOX2 N terminal 5'-CGGGCCCGCAGCAAACTTCG GGG, SOX2 C terminal 5'-CGGCCCTCACATGTGTGAGA GGG. SFTPC 5'-GCGTCCTAGATGTAGTAGAG CGG. TP63 5'-TGATGCGCTGTTGCTTATTG CGG.
\end{abstract}

PAM sequences are underlined.

\section{Human foetal intestinal culture and generation of SOX9 reporter organoids}

Human foetal gut samples at around 9- to 11-week gestation were obtained with ethical approval (REC-96/085) following maternal consent. Human foetal intestinal organoids were generated from the ileum of the tissue and maintained as previously described (Ross et al., 2021). Briefly, the human foetal intestine tissue was placed in a six-well plate and dissociated in a Hank's Balanced Salt Solution (HBSS) medium (14170088, Thermo Fisher Scientific) containing 1.07 Wunsch units/ $\mathrm{ml}$ of Liberase DH (5401054001, Merck) on a shaking platform at $750 \mathrm{rpm}$ in a $37^{\circ} \mathrm{C}$ incubator for $15 \mathrm{~min}$. The sample was then mechanically disrupted by pipetting up and down with a P1000 pipette, transferred to an Eppendorf tube and centrifuged for $5 \mathrm{~min}$ at $400 \mathrm{~g}$. The supernatant was removed and the pellet was washed three times with Dulbecco's Modified Eagle Medium/F12 (DMEM/F12, 11320033, Thermo Fisher Scientific) by centrifugation. Later, the pellet was re-suspended in Matrigel (Corning, 356231) and seeded in a 48-well plate. The plate was incubated at $37^{\circ} \mathrm{C}$ for $5 \mathrm{~min}$ to solidify the Matrigel and $250 \mu \mathrm{l}$ of self-renewing medium were added. The self-renewing medium for human foetal intestinal organoids contains Wnt3a conditioned medium 
(50\%, v/v, Stem Cell Institute, University of Cambridge), R-spondin-1 conditioned medium (20\% v/v, Stem Cell Institute, University of Cambridge), Primocin (500 $\mu \mathrm{g} / \mathrm{mL}$, ant-pm-2, InvivoGen), B27 (1:50, 17504044, Thermo Fisher Scientific), nicotinamide (10 mM, N3376, Merck), N-acetylcysteine (1.25 mM, A9165, Merck), A3801 (500 nM, 2939, Tocris), SB202190 (10 „ M, S7067, Merck), Murine EGF (50 ng/mL, PMG8041, Thermo Fisher Scientific) and Murine Noggin (100 ng/mL, 250-38, PeproTech). The medium was replaced every 2-3 days and organoids were passaged by mechanical disruption with a P1000 pipette and re-seeded in Matrigel with fresh self-renewing medium every 7-10 days.

For gene targeting, $3 \mu \mathrm{l}$ spCas9 $(4 \mu \mathrm{g} / \mu \mathrm{l})$ and $4.32 \mu \mathrm{l}$ of SOX9 ssRNA (100 $\mu \mathrm{M}$, Synthego) were mixed and incubated at RT for a minimum of $10 \mathrm{~min}$ in order to form ssRNPs. At the same time, human foetal intestinal organoids were dissociated into single cells, according to the protocol previously described for nucleofection. $6 \times 10^{5}$ cells were suspended using Lonza Nucleofection P3 buffer, mixed with $6 \mu \mathrm{g}$ of SOX9 reporter repair template plasmid. The cell suspension was further mixed with preformed Cas9 RNPs and equally distributed into three $20 \mu$ cuvettes. Nucleofection was performed using the program EA125 for human intestinal lung organoids. After nucleofection, intestinal organoid maintenance medium with ROCKi was added to dilute the P3 buffer. The cell mixture was then taken out and seeded in Matrigel in six wells of a 24-well plate and cultured with maintenance medium with $10 \mu \mathrm{M}$ ROCKi for 1 week before flow cytometry.

EGFP $^{+}$cells were sorted, harvested and pooled together in $1 \times 24$-well plate and cultured with intestinal organoid maintenance medium with ROCKi before organoid colonies formed. Individual organoid colonies were picked based on the expression of EGFP observed using EVOS FL system (Thermo Fisher Scientific) microscope and seeded in a 48-well plate to successfully expand them as organoid lines. These organoid lines were then harvested for genomic DNA isolation and genotyping.

\section{Small-molecule influence on gene targeting efficiency}

mEGFP-ACTB gene targeting was performed as previously described. After nucleofection, DMSO

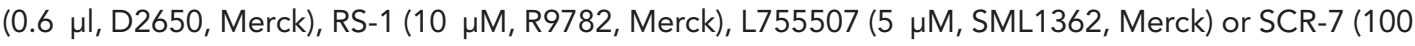
$\mu \mathrm{M}, \mathrm{SML1546}$, Merck) were added to self-renewing medium with ROCKi for $48 \mathrm{hr}$. Organoid cells were analysed by flow cytometry $72 \mathrm{hr}$ after nucleofection.

\section{T7 endonuclease assay}

To test for site-specific DNA cleavage using the T7 endonuclease assay, organoid cells were harvested $48 \mathrm{hr}$ after nucleofection of ssRNP, tr/cr RNP, plasmid encoding Cas9 and gRNA or WT control organoids. Genomic DNA was extracted using QIAamp Fast DNA Tissue Kit (51404, Qiagen). PCR was performed using PrimeSTAR GXL DNA Polymerase (R050A, Takara) with 20 ng of genomic DNA as template according to the manufacturer's protocol. Forward primer 5'-TTGCCAATGGGGATCGCAG-3' and reverse primer 5'-GCTCGATGGGGTACTTCAGG-3' were used for ACTB locus amplification. $10 \mu \mathrm{l}$ of PCR product was then mixed with $1.5 \mu \mathrm{l} 10 \mathrm{X}$ NEBuffer 2 (B7002S, NEB) and $1.5 \mu \mathrm{l}$ of nuclease-free water. The mixture was denatured at $95^{\circ} \mathrm{C}$ for $10 \mathrm{~min}$, followed by ramp $-2^{\circ} \mathrm{C} / \mathrm{s} 95^{\circ} \mathrm{C}$ to $85^{\circ} \mathrm{C}$ and ramp $-0.3^{\circ} \mathrm{C} / \mathrm{s}$ from $85^{\circ} \mathrm{C}$ to $25^{\circ} \mathrm{C}$. $2 \mu \mathrm{l} \mathrm{T7}$ Endonu-

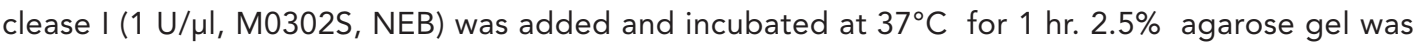
used for electrophoresis.

\section{ICE analysis for indel production}

Genomic DNA was extracted from organoid cells which were harvested $48 \mathrm{hr}$ after nucleofection of ssRNP, tr/cr RNP, plasmid encoding Cas9 and gRNA or WT control organoids using QIAamp Fast DNA Tissue Kit (51404, Qiagen). PCR was performed using PrimeSTAR GXL DNA Polymerase (R050A, Takara) with 20 ng of genomic DNA as template according to the manufacturer's protocol. Forward primer 5'-TTGCCAATGGGGATCGCAG-3' and reverse primer 5'-GCTCGATGGGGTACTTCAGG-3' were used for ACTB locus amplification. PCR products were cleaned up using Macherey-Nagel NucleoSpin Gel and PCR Clean-up Kit (Macherey-Nagel, 740609.50) and sent for Sanger Sequencing (Department of Biochemistry, University of Cambridge) using reverse primer 5'-GCTCGATGGGGTACTTCAGG-3'. Sanger sequencing results were compared using ICE online CRISPR editing analysis tool: https://www.synthego.com/products/bioinformatics/crispr-analysis. 


\section{Flow cytometry analysis}

Organoid single cells were prepared $72 \mathrm{hr}$ after nucleofection, lentivirus transduction or Lipofectamine transfection. Cells were analysed using Sony SH800S Cell Sorter and FlowJo software (version 10.4).

\section{Organoid genotyping}

Organoids from a single 48-well plate well were used for genomic DNA extraction with QIAamp Fast DNA Tissue Kit (51404, Qiagen) according to the manufacturer's protocol. PCR was performed using PrimeSTAR GXL DNA Polymerase (R050A, Takara) with 20 ng of genomic DNA as template according to the manufacturer's protocol. Primers used are listed in Supplementary file 2. For each gene targeting, three randomly picked lines were chosen for further Sanger sequencing. 5' and 3' homologous arms of the gene targeting product were amplified using PrimeSTAR GXL DNA Polymerase with the aforementioned primers. PCR products were cleaned up using Macherey-Nagel NucleoSpin Gel and PCR Clean-up Kit (Macherey-Nagel, 740609.50) and sequenced using Sanger sequencing (Department of Biochemistry, University of Cambridge).

\section{Evaluation of off-target effects}

SOX9 gRNA potential off-target sites were identified using the IDT online service: https://eu.idtdna. $\mathrm{com} / \mathrm{site} /$ order/designtool/index/CRISPR_SEQUENCE. We selected nine potential off-target sites with the least mismatches (three mismatches) from all off-target sites with NGG PAM. Primers (Supplementary file 2) were designed to amplify the flanking regions of these sites, purified and sent for Sanger sequencing.

\section{Alveolar differentiation of lung organoids}

For alveolar differentiation, organoids were transferred to 300 Pa stiffness poly-ethylene-glycol (PEG) mixture containing RGD adhesion peptides synthesised in Prof. Matthias Lutolf's lab as previously published (Gjorevski and Lutolf, 2017). Alveolar differentiation medium, containing N2 (1:100, Thermo Fisher Scientific, 17502-048), B27 (1:50, Thermo Fisher Scientific, 12587-010), N-acetylcysteine (1.25 mM, Merck, A9165), FGF10 (100 ng/ml, PeproTech, 100-26), FGF7 (100 ng/ml, PeproTech, 100-19), CHIR99021 (3 $\mu \mathrm{M}$, Stem Cell Institute, University of Cambridge), dexamethasone (50 nM, Merck, D4902), cAMP (0.1 mM, Merck, B5386), IBMX (0.1 mM, Merck, I5879) and DAPT (50 $\mu$ M, Merck, D5942) was used for 6 days.

\section{Western blot}

Organoids were harvested, washed twice with Advanced DMEM/F12 and then twice with PBS before pelleting at $300 \mathrm{~g}$ for $5 \mathrm{~min}$. Organoid cell pellets were re-suspended in 100-200 $\mu$ of RIPA buffer (Merck, R0278) with protease inhibitor (Thermo Fisher Scientific, 87786) added. Organoid suspension was incubated for $30 \mathrm{~min}$ on ice, with strong vortex every $5 \mathrm{~min}$. Cell pieces and debris were removed by centrifugation at 13,000 rpm. Supernatant was harvested. Protein concentration was measured by BCA assay (Thermo Fisher Scientific, 23227). Equal amount of each protein sample was mixed with Sample Buffer (Bio-Rad, 1610747) and beta-mercaptoethanol according to the manufacturer's protocol. Mixture was heated at $95^{\circ} \mathrm{C}$ for $5 \mathrm{~min}$ and cooled down to RT.

Samples were then separated on a 4-12\% SDS-PAGE and transferred to nitrocellulose membranes. Membrane was blocked by PBS with 5\% BSA for $1 \mathrm{hr}$ at RT. Proteins were detected by incubation with primary antibodies (SOX2, Bio-Techne, AF2018, 1:1000, RRID:AB_355110; $\alpha$-Tubulin, Merck, T6199, 1:2000, RRID:AB_477583; GFP, Abcam, ab13970, 1:5000, RRID:AB_300798; $\beta$-actin, Merck, A3854, 1:50,000, RRID:AB_262011) and secondary antibodies (Donkey anti-Goat IRDye 800CW, Abcam, ab216775, 1:1000, RRID:AB_2893338; Donkey anti-Mouse IRDye 800CW, ab216774, 1:1000, RRID:AB_2893339; IRDye 680LT Donkey anti-Chicken Secondary Antibody, Li-Cor, 925-68028, 1:20,000, RRID:AB_2814923). Protein bands were visualised using Li-Cor Odyssey system.

\section{Lentivirus infection of organoids for inducible knockdown and activation}

Organoids were dissociated into small organoid pieces or single cells using pre-warmed TrypLE Express at $37^{\circ} \mathrm{C}$ for $5-10 \mathrm{~min}$. Organoid cells were then spun down at $300 \mathrm{~g}$ for $5 \mathrm{~min}$. The cell pellet of one 24-well plate was re-suspended in $500 \mu$ self-renewing medium with ROCKi. $20 \mu$ of inducible 
CRISPRi or CRISPRa lentivirus was added and mixed well. The mixture was incubated at $37^{\circ} \mathrm{C}$ overnight. The next morning, organoid cells were pelleted at $300 \mathrm{~g}$ for $5 \mathrm{~min}$, washed twice with PBS and seeded in $100 \mu \mathrm{l}$ Matrigel into two wells of a 24-well plate. Organoid cells were cultured with self-renewing medium with ROCKi for 3 days before dissociating with TrypLE Express into single cells for cell sorting. TagRFP ${ }^{+}$cells were sorted using Sony SH800S Cell Sorter and pooled together and seeded in Matrigel at 3000-5000 cells/well of a 24-well plate. Organoid cells were then expanded for around 17 days using self-renewing medium with ROCKi. At this stage, organoids with inducible CRISPRi and CRISPRa system could be frozen as parental lines.

Organoids with inducible CRISPRi and CRISPRa system were broken into small organoid pieces, or single cells, similarly and transduced with gRNA lentivirus (5-10 $\mu$ l virus/500 $\mu$ l organoid cell re-suspension). The mixture was incubated overnight, and the next morning organoid cells were pelleted, washed and seeded with Matrigel as described above. After 3 days of culturing with self-renewing medium supplemented with ROCKi, TagRFP and EGFP double-positive cells were sorted and seeded in Matrigel at $\sim 2000-3000$ cells/well of a 24-well plate and expanded for 10-17 days before turning on inducible CRISPRa and CRISPRi. Dox (2 $\mathrm{gg} / \mathrm{ml}$, Merck, D9891) and TMP (10 nmol/l, Merck, 92131) were supplemented in self-renewing medium accordingly. Medium was refreshed every $48 \mathrm{hr}$.

For evaluation of inducible CRISPRi using CD71 as target, single cells were prepared as described above after 5 days of Dox and TMP treatment. Cells were pelleted at $300 \mathrm{~g}$ for $5 \mathrm{~min}$ and re-suspended in $100 \mu \mathrm{l}$ PBS with 0.5\% BSA and 2 mM EDTA. $2.5 \mu \mathrm{l}$ of PE/Cy7 anti-human CD71 antibody (BioLegend, 334111, RRID:AB_2563118) was added and incubated at $4^{\circ} \mathrm{C}$ for $30 \mathrm{~min}$. Cells were then washed with PBS with $0.5 \%$ BSA and 2 mM EDTA twice and re-suspended in $300 \mu$ l of PBS with $0.5 \%$ BSA and 2 mM EDTA for flow cytometry analysis.

\section{RNA extraction, CDNA synthesis and qRT-PCR}

Organoids were harvested and washed twice with Advanced DMEM/F12, before $350 \mu$ of RLT buffer was added to lyse organoids. RNA extraction was performed according to the manufacturer's protocol using RNeasy Mini Kit (Qiagen, 74104) with RNase-Free DNase Set (Qiagen, 79254). RNA concentrations were measured by Nanodrop (Thermo Fisher Scientific). cDNA was synthesised with MultiScribe Reverse Transcriptase (Thermo Fisher Scientific, 4311235) according to the manufacturer's protocol. cDNA was diluted 1:25 and $6 \mu$ l was used for one qPCR reaction with PowerUp SYBR Green Master Mix (Thermo Fisher Scientific, A25776). Relative gene expression was calculated using the $\triangle \triangle C T$ method relative to $A C T B$ control.

\section{Plasmid construction}

eSpCas9(1.1) was a gift from Feng Zhang (Addgene plasmid \# 71814). ACTB gRNA sequence 5'-GCTATTCTCGCAGCTCACC-3' was cloned into the vector using Bbsl sites. ACTB repair template AICSDP-15: ACTB-mEGFP was a gift from The Allen Institute for Cell Science (Addgene plasmid \# 87425). SOX9 repair template was created by Infusion (638909, Takara) cloning to insert SOX9 5' and 3' homologous arms in EasyFusion T2A-H2B-GFP plasmid (a gift from Janet Rossant, Addgene plasmid \# 112851). AAVS1 repair template was created by Infusion cloning to swap the CAG promoter and Puromycin resistance cassette in plasmid AICSDP-42: AAVS1-mTagRFPT-CAAX (a gift from The Allen Institute for Cell Science, Addgene plasmid \# 107580). SOX2 KO repair template was created by Infusion cloning to insert SOX2 5' and 3' homologous arms in EasyFusion T2A-H2B-GFP (a gift from Janet Rossant, Addgene plasmid \# 112851). SFTPC targeting repair template was created by Infusion assembly of SFTPC 5' and 3' homologous arms together with T2A-Rox-EF1a-Rox-Venus-NLS. TP63 targeting repair template was created by Infusion assembly of TP63 5' and $3^{\prime}$ homologous arms together with T2A-Rox-EF1a-Rox-Venus-NLS. CMV-Dre-T2A-TagRFP vector was created by Infusion assembly of Dre (a gift from Azim Surani Group, Gurdon Institute, Cambridge) and T2A-TagRFP sequences together. NKX2-1 overexpression vector was created by inserting EF1a-TagRFP-2A-tet3G and tetON-NKX2-1 CDS in two steps cloning using Infusion cloning into a pHAGE backbone. The minimal CMV-GFP(1-10) plasmid was created by Infusion cloning of CMV-GFP(1-10) from pcDNA3.1-GFP(1-10) (a gift from Bo Huang, Addgene plasmid \# 70219) into a pUC57 backbone. For testing lentiviral transduction efficiency, CMV-myrAKT-IRES-GFP vector was created by Infusion cloning to insert myrAKT from pCCL-Akt1 (a gift from Bi-Sen Ding, Icahn School of Medicine, Mount Sinai) and IRES sequence into pFP945 (a gift from Frederick Livesey, University College London). The Dox-inducible CRISPRi vector 
was created by sub-cloning N-terminal KRAB-dCas9 (a gift from Bruce Conklin, Addgene plasmid \# 73498) into the NKX2-1 overexpressing vector using Xhol and BamHI sites. Dox-inducible CRISPRi with DD control vector was created by In-fusion cloning of a C-terminal DHFR sequence into the Doxinducible CRISPRi vector using BamHI site. The inducible CRISPRa vector was created by sub-cloning dxCas9(3.7)-VPR (a gift from David Liu, Addgene plasmid \# 108383) into the NKX2-1 overexpressing vector using Xhol and BamHI sites. The gRNA entry vector was cloned by infusion cloning of a EF1a promoter into pKLV2-U6gRNA5(Bbsl)-PGKpuro2ABFP-W vector (a gift from Kosuke Yusa, Addgene plasmid \# 67974) using BamHI and EcoRI sites, and then cloned a EGFP-CAAX to swap the EGFP sequence using Xhol and Notl sites. All plasmids have been deposited in Addgene: https://www. addgene.org/browse/article/28216212/.

\section{Acknowledgements}

We thank Dr. Andrew Bassett (Wellcome Sanger Institute) for the Cas9 expressing vector. We thank Prof. Luke Gilbert (UCSF), Prof. Martin Kampmann (UCSF) and Prof. Ruilin Tian (SUSTech) for their advice on CRISPRi and CRISPRa. We would like to acknowledge the Gurdon Institute Imaging Facility for microscopy support.

DS is supported by a Wellcome Trust PhD studentship (109146/Z/15/Z) and the Department of Pathology, University of Cambridge. LDE is supported by the Alzheimer's Research UK Stem Cell Research Centre, funded by the Alborada Trust. VS is supported by a Wellcome Trust PhD studentship (102175/B/13/Z). KL is supported by Basic Science Research Program through the National Research Foundation of Korea (NRF) funded by the Ministry of Education (2018R1A6A3A03012122). MZ was funded by a Medical Research Council (MRC) New Investigator Research Grant (MR/T001917/1). ELR is supported by Medical Research Council (MR/P009581/1). Core support from the Wellcome Trust (203144/Z/16/Z) and Cancer Research UK (C6946/A24843).

\section{Additional information}

Funding

\begin{tabular}{|c|c|c|}
\hline Funder & Grant reference number & Author \\
\hline Medical Research Council & MR/P009581/1 & Emma L Rawlins \\
\hline Wellcome Trust & $\begin{array}{l}\text { PhD Studentship } \\
\text { 109146/Z/15/Z }\end{array}$ & Dawei Sun \\
\hline $\begin{array}{l}\text { Alzheimers Research UK } \\
\text { Stem Cell Research Centre }\end{array}$ & & Lewis Evans \\
\hline $\begin{array}{l}\text { National Research } \\
\text { Foundation of Korea }\end{array}$ & 2018R1A6A3A03012122 & Kyungtae Lim \\
\hline Wellcome Trust & $\begin{array}{l}\text { Core Support for Gurdon } \\
\text { Institute 203144/Z/16/Z }\end{array}$ & Emma L Rawlins \\
\hline Cancer Research UK & $\begin{array}{l}\text { Core Support for Gurdon } \\
\text { Institute C6946/A24843 }\end{array}$ & Emma L Rawlins \\
\hline Medical Research Council & $\begin{array}{l}\text { New Investigator Research } \\
\text { Grant MR/T001917/1 }\end{array}$ & Matthias Zilbauer \\
\hline Wellcome Trust & $\begin{array}{l}\text { PhD studentship } \\
\text { 102175/B/13/Z }\end{array}$ & Vanesa Sokleva \\
\hline
\end{tabular}

The funders had no role in study design, data collection and interpretation, or the decision to submit the work for publication.

Author contributions

Dawei Sun, Conceptualization, Formal analysis, Investigation, Writing - original draft, Writing review and editing; Lewis Evans, Kyungtae Lim, Investigation, Writing - review and editing; Francesca Perrone, Investigation, Resources, Validation; Vanesa Sokleva, Investigation, Validation; Saba 
Rezakhani, Matthias Lutolf, Methodology, Resources; Matthias Zilbauer, Supervision; Emma L Rawlins, Conceptualization, Funding acquisition, Project administration, Supervision, Writing - original draft, Writing - review and editing

Author ORCIDs

Dawei Sun (iD http://orcid.org/0000-0003-1551-4349

Lewis Evans (iD http://orcid.org/0000-0001-7279-7651

Kyungtae Lim (iD http://orcid.org/0000-0001-6044-2191

Emma L Rawlins (DiD http://orcid.org/0000-0001-7426-3792

Decision letter and Author response

Decision letter https://doi.org/10.7554/eLife.67886.sa1

Author response https://doi.org/10.7554/eLife.67886.sa2

\section{Additional files}

Supplementary files

- Transparent reporting form

- Supplementary file 1. Summary of genetic manipulation efficiency in different organoid lines.

- Supplementary file 2. Primers and gRNA sequences used in this study.

- Supplementary file 3. Raw quantification data. Summary of qRT-PCR results, flow cytometry quantification results of DNA delivery efficiency, small-molecule function and CRISPRi kinetics analysis and ICE analysis results.

- Source data 1. Gels and blots.

Data availability

All data generated or analysed during this study are included in the manuscript and supporting files.

\section{References}

Anastassiadis K, Fu J, Patsch C, Hu S, Weidlich S, Duerschke K, Buchholz F, Edenhofer F, Stewart AF. 2009. Dre recombinase, like $\mathrm{Cre}$, is a highly efficient site-specific recombinase in $E$. coli, mammalian cells and mice. Disease Models \& Mechanisms 2: 508-515. DOI: https://doi.org/10.1242/dmm.003087, PMID: 19692579

Artegiani B, Hendriks D, Beumer J, Kok R, Zheng X, Joore I, Chuva de Sousa Lopes S, van Zon J, Tans S, Clevers H. 2020. Fast and efficient generation of knock-in human organoids using homology-independent CRISPR-Cas9 precision genome editing. Nature Cell Biology 22: 321-331. DOI: https://doi.org/10.1038/ s41556-020-0472-5, PMID: 32123335

Barkauskas CE, Cronce MJ, Rackley CR, Bowie EJ, Keene DR, Stripp BR, Randell SH, Noble PW, Hogan BLM. 2013. Type 2 alveolar cells are stem cells in adult lung. The Journal of Clinical Investigation 123: 3025-3036. DOI: https://doi.org/10.1172/JCl68782, PMID: 23921127

Bowden AR, Morales-Juarez DA, Sczaniecka-Clift M, Agudo MM, Lukashchuk N, Thomas JC, Jackson SP. 2020. Parallel CRISPR-Cas9 screens clarify impacts of p53 on screen performance. eLife 9: e55325. DOI: https://doi. org/10.7554/eLife.55325, PMID: 32441252

Bruntraeger M, Byrne M, Long K, Bassett AR. 1961. Editing the Genome of Human Induced Pluripotent Stem Cells Using CRISPR/Cas9 Ribonucleoprotein Complexes. Methods in Molecular Biology 1: 153-183. DOI: https://doi.org/10.1007/978-1-4939-9170-9_11

Elmentaite R, Ross ADB, Roberts K, James KR, Ortmann D, Gomes T, Nayak K, Tuck L, Pritchard S, Bayraktar OA, Heuschkel R, Vallier L, Teichmann SA, Zilbauer M. 2020. Single-Cell Sequencing of Developing Human Gut Reveals Transcriptional Links to Childhood Crohn's Disease. Developmental Cell 55: 771-783. DOI: https://doi.org/10.1016/j.devcel.2020.11.010, PMID: 33290721

Gilbert LA, Larson MH, Morsut L, Liu Z, Brar GA, Torres SE, Stern-Ginossar N, Brandman O, Whitehead EH, Doudna JA, Lim WA, Weissman JS, Qi LS. 2013. CRISPR-mediated modular RNA-guided regulation of transcription in eukaryotes. Cell 154: 442-451. DOl: https://doi.org/10.1016/j.cell.2013.06.044, PMID: 23849981

Gjorevski N, Sachs N, Manfrin A, Giger S, Bragina ME, Ordóñez-Morán P, Clevers H, Lutolf MP. 2016. Designer matrices for intestinal stem cell and organoid culture. Nature 539: 560-564. DOI: https://doi.org/10.1038/ nature20168, PMID: 27851739

Gjorevski N, Lutolf MP. 2017. Synthesis and characterization of well-defined hydrogel matrices and their application to intestinal stem cell and organoid culture. Nature Protocols 12: 2263-2274. DOI: https://doi.org/ 10.1038/nprot.2017.095, PMID: 28981121 
Horlbeck MA, Gilbert LA, Villalta JE, Adamson B, Pak RA, Chen Y, Fields AP, Park CY, Corn JE, Kampmann M, Weissman JS. 2016. Compact and highly active next-generation libraries for CRISPR-mediated gene repression and activation. eLife 5: e19760. DOI: https://doi.org/10.7554/eLife.19760, PMID: 27661255

Hu JH, Miller SM, Geurts MH, Tang W, Chen L, Sun N, Zeina CM, Gao X, Rees HA, Lin Z, Liu DR. 2018. Evolved Cas9 variants with broad PAM compatibility and high DNA specificity. Nature 556: 57-63. DOI: https://doi.org/ 10.1038/nature26155, PMID: 29512652

Iwamoto M, Björklund T, Lundberg C, Kirik D, Wandless TJ. 2010. A General Chemical Method to Regulate Protein Stability in the Mammalian Central Nervous System. Chemistry \& Biology 17: 981-988. DOI: https:// doi.org/10.1016/j.chembiol.2010.07.009, PMID: 20851347

Kim S, Kim D, Cho SW, Kim J, Kim JS. 2014. Highly efficient RNA-guided genome editing in human cells via delivery of purified Cas9 ribonucleoproteins. Genome Research 24: 1012-1019. DOI: https://doi.org/10.1101/ gr.171322.113, PMID: 24696461

Lim K, Tang W, Sun D, He P, Teichmann SA, Marioni JC, Meyer KB, Rawlins EL. 2021. Acquisition of Alveolar Fate and Differentiation Competence by Human Fetal Lung Epithelial Progenitor Cells. [bioRxiv]. DOI: https://doi. org/10.1101/2021.06.30.450501

Lin S, Staahl BT, Alla RK, Doudna JA. 2014. Enhanced homology-directed human genome engineering by controlled timing of CRISPR/Cas9 delivery. eLife 3: e04766. DOI: https://doi.org/10.7554/eLife.04766, PMID: 25497837

Mandegar MA, Huebsch N, Frolov EB, Shin E, Truong A, Olvera MP, Chan AH, Miyaoka Y, Holmes K, Spencer Cl, Judge LM, Gordon DE, Eskildsen TV, Villalta JE, Horlbeck MA, Gilbert LA, Krogan NJ, Sheikh SP, Weissman JS, Qi LS, et al. 2016. CRISPR Interference Efficiently Induces Specific and Reversible Gene Silencing in Human iPSCs. Cell Stem Cell 18: 541-553. DOI: https://doi.org/10.1016/j.stem.2016.01.022, PMID: 26971820

Maruyama T, Dougan SK, Truttmann MC, Bilate AM, Ingram JR, Ploegh HL. 2015. creasing the efficiency of precise genome editing with CRISPR-Cas9 by inhibition of nonhomologous end joining. Nature Biotechnology 33: 538-542. DOI: https://doi.org/10.1038/nbt.3190, PMID: 25798939

Nikolić MZ, Caritg O, Jeng Q, Johnson JA, Sun D, Howell KJ, Brady JL, Laresgoiti U, Allen G, Butler R, Zilbauer M, Giangreco A, Rawlins EL. 2017. Human embryonic lung epithelial tips are multipotent progenitors that can be expanded in vitro as long-term self-renewing organoids. eLife 6: e26575. DOI: https://doi.org/10. 7554/eLife.26575, PMID: 28665271

Nuñez JK, Chen J, Pommier GC, Cogan JZ, Replogle JM, Adriaens C, Ramadoss GN, Shi Q, Hung KL, Samelson AJ, Pogson AN, Kim JYS, Chung A, Leonetti MD, Chang HY, Kampmann M, Bernstein BE, Hovestadt V, Gilbert LA, Weissman JS. 2021. Genome-wide programmable transcriptional memory by CRISPR-based epigenome editing. Cell 184: 2503-2519. DOI: https://doi.org/10.1016/j.cell.2021.03.025, PMID: 33838111

Paquet D, Kwart D, Chen A, Sproul A, Jacob S, Teo S, Olsen KM, Gregg A, Noggle S, Tessier-Lavigne M. 2016. Efficient introduction of specific homozygous and heterozygous mutations using CRISPR/Cas9. Nature 533: 125-129. DOI: https://doi.org/10.1038/nature17664, PMID: 27120160

Roberts B, Haupt A, Tucker A, Grancharova T, Arakaki J, Fuqua MA, Nelson A, Hookway C, Ludmann SA, Mueller IA, Yang R, Horwitz R, Rafelski SM, Gunawardane RN. 2017. Systematic gene tagging using CRISPR/ Cas9 in human stem cells to illuminate cell organization. Molecular Biology of the Cell 28: 2854-2874. DOI: https://doi.org/10.1091/mbc.e17-03-0209, PMID: 28814507

Roberts B, Hendershott MC, Arakaki J, Gerbin KA, Malik H, Nelson A, Gehring J, Hookway C, Ludmann SA, Yang R, Haupt A, Grancharova T, Valencia V, Fuqua MA, Tucker A, Rafelski SM, Gunawardane RN. 2019. Fluorescent Gene Tagging of Transcriptionally Silent Genes in hiPSCs. Stem Cell Reports 12: 1145-1158. DOI: https://doi.org/10.1016/j.stemcr.2019.03.001, PMID: 30956114

Rock JR, Onaitis MW, Rawlins EL, Lu Y, Clark CP, Xue Y, Randell SH, Hogan BLM. 2009. Basal cells as stem cells of the mouse trachea and human airway epithelium. PNAS 106: 12771-12775. DOI: https://doi.org/10.1073/pnas. 0906850106, PMID: 19625615

Ross ADB, Perrone F, Elmentaite R, Teichmann SA, Zilbauer M. 2021. Obtaining purified human intestinal epithelia for single-cell analysis and organoid culture. STAR Protocols 2: 100597. DOI: https://doi.org/10.1016/ j.xpro.2021.100597, PMID: 34169291

Smith JR, Maguire S, Davis LA, Alexander M, Yang F, Chandran S, ffrench-Constant C, Pedersen RA. 2008. Robust, persistent transgene expression in human embryonic stem cells is achieved with AAVS1-targeted integration. Stem Cells 26: 496-504. DOI: https://doi.org/10.1634/stemcells.2007-0039, PMID: 18024421

Smits AH, Ziebell F, Joberty G, Zinn N, Mueller WF, Clauder-Münster S, Eberhard D, Fälth Savitski M, Grandi P, Jakob P, Michon AM, Sun H, Tessmer K, Bürckstümmer T, Bantscheff M, Steinmetz LM, Drewes G, Huber W. 2019. Biological plasticity rescues target activity in CRISPR knock outs. Nature Methods 16: 1087-1093. DOI: https://doi.org/10.1038/s41592-019-0614-5, PMID: 31659326

Song J, Yang D, Xu J, Zhu T, Chen YE, Zhang J. 2016. RS-1 enhances CRISPR/Cas9- and TALEN-mediated knock-in efficiency. Nature Communications 7: 1-7. DOI: https://doi.org/10.1038/ncomms10548, PMID: 26817820

Tian R, Gachechiladze MA, Ludwig CH, Laurie MT, Hong JY, Nathaniel D, Prabhu AV, Fernandopulle MS, Patel R, Abshari M, Ward ME, Kampmann M. 2019. CRISPR Interference-Based Platform for Multimodal Genetic Screens in Human iPSC-Derived Neurons. Neuron 104: 239-255. DOI: https://doi.org/10.1016/j.neuron.2019. 07.014, PMID: 31422865

Tuladhar R, Yeu Y, Tyler Piazza J, Tan Z, Rene Clemenceau J, Wu X, Barrett Q, Herbert J, Mathews DH, Kim J, Hyun Hwang T, Lum L. 2019. CRISPR-Cas9-based mutagenesis frequently provokes on-target mRNA 
misregulation. Nature Communications 10: 1-10. DOI: https://doi.org/10.1038/s41467-019-12028-5, PMID: 31492834

Yao X, Zhang M, Wang X, Ying W, Hu X, Dai P, Meng F, Shi L, Sun Y, Yao N, Zhong W, Li Y, Wu K, Li W, Chen ZJ, Yang H. 2018. Tild-CRISPR Allows for Efficient and Precise Gene Knockin in Mouse and Human Cells.

Developmental Cell 45: 526-536. DOl: https://doi.org/10.1016/j.devcel.2018.04.021, PMID: 29787711

Yu C, Liu Y, Ma T, Liu K, Xu S, Zhang Y, Liu H, La Russa M, Xie M, Ding S, Qi LS. 2015. Small Molecules Enhance CRISPR Genome Editing in Pluripotent Stem Cells. Cell Stem Cell 16: 142-147. DOI: https://doi.org/10.1016/j. stem.2015.01.003, PMID: 25658371 